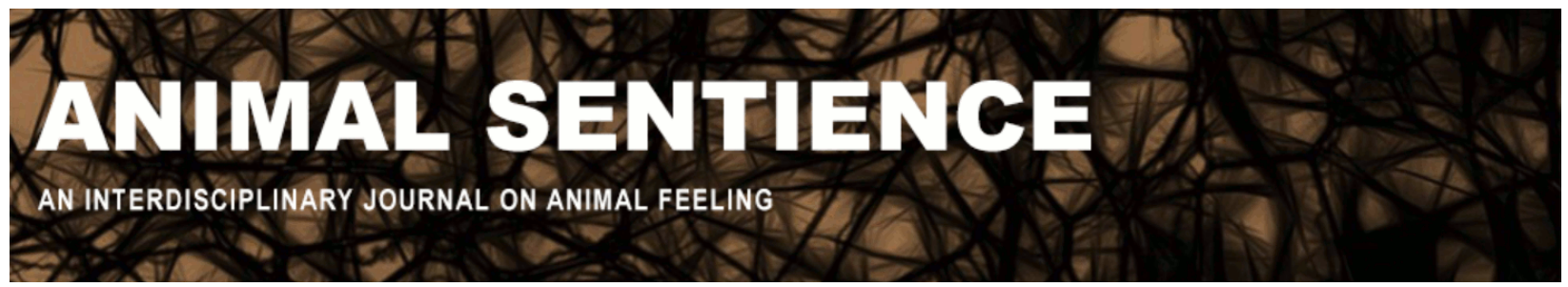

Signal, Tania (2016) When the client is not the abuser, but one of the abused. Animal Sentience 6(3)

DOI: $10.51291 / 2377-7478.1089$

Date of submission: 2016-02-17

Date of acceptance: 2016-02-22

(c) 


\title{
When the client is not the abuser, but one of the abused
}

Commentary on Lachance on Breaking Silence

\author{
Tania Signal \\ Department of Psychology \\ Central Queensland University, Australia
}

\begin{abstract}
The question of client confidentiality and reporting animal abuse is complicated when the client is not the abuser, and when the abuse (of both people and animals) may escalate precisely because it has been (or may be) reported.
\end{abstract}

Tania Signal t.signal@cqu.edu.au,
Associate Professor of Psychology,
cQUniversity, studies Human-Animal
Interaction: potential links between
personality factors (especially empathy),
interpersonal violence and the
treatment of animals, as well as
community attitudes to farm animal welfare and suitable
penalties for animal cruelty offenses.
https://www.cqu.edu.au/about-us/staff-
directory/profiles/school-of-human,-health-and-social-
sciences/school-of-human,-health-and-social-
sciences/signalt

A number of points could be made in response to Lachance's (2016) important paper. These include the socio-legal implications if vets don't report (e.g., Robertson, 2010) and concerns (similar to those about suspected child abuse) that veterinary care won't be sought for abused animals if reporting is mandatory. Some of these points have been raised recently (e.g., Acutt, Signal \& Taylor, 2015) so I will not repeat them again here.

What I would like to focus on is the emphasis the target article places on situations where the perpetrator brings in an abused animal. Although Lachance does briefly present two studies that acknowledge the overlap between animal abuse and domestic/family violence, this fails to capture the extent of the role that animals can and do play in the lives of abused women. ${ }^{1}$ The literature suggests that a much more likely scenario is one where a veterinarian is faced with a case of suspected animal abuse when the person who brings the animal in may also be a victim of violence. This situation has implications across legal, ethical and moral domains very different from the one where the abuser brings the animal in.

Research suggests that the prevalence of companion animal abuse within the milieu of domestic/family violence is relatively similar across cultures and countries. Of abused

\footnotetext{
${ }^{1}$ This is not to suggest that companion animal abuse only occurs in heterosexual relationships where women (and children) are the target of violence. However, a significant research gap exists when it comes to examining companion animal abuse within same-sex relationships or in situations where the male partner is the target of violence.
} 
women (who have pets), $40-75 \%$ report threats or actual harm to their companion animals at the hands of their abuser (e.g., Flynn, 2011). Abusive relationships have many victims, including children. (For a review of the effects of witnessing animal abuse and its correlates with other forms of child abuse see Becker \& French, 2004.) But even when we consider only the adults in the abusive relationship, abusers who also harm or kill companion animals commit more psychological aggression and physical assault, including emotional and sexual violence, stalking and controlling behaviour than those who do not harm animals (e.g., Simmons \& Lehmann, 2007; Febres et al., 2014). Women may also delay their own escape from violent relationships when they are unable to take their animals with them into refuge with the attendant risk of escalating violence toward both humans and animals (e.g., Hardesty, Khaw, Ridgway, Weber \& Miles, 2013; Taylor, Signal \& Stark, 2006).

Thus, besides its obvious importance for companion animal welfare, positive identification by a veterinarian of deliberate animal harm may assist other vulnerable members of the "family," including children and other adults. The sensitive nature of discussing the possibility of family violence with a client, however, and the risk of escalating the violence if a report or investigation is initiated raise issues beyond those noted in the target article.

Two studies of women in a violent relationship who had experienced both personal abuse and deliberate harm to their companion animals found that although the majority of the women had sought veterinary care for their animals there was a mixed response to the possibility of disclosing family violence to the attending veterinarian. Hardesty, Khaw, Ridgway, Weber and Miles (2013) reported that just over half of their participants felt it would have been acceptable for veterinarians to screen for family violence, but only in situations where the abuser was not present. In contrast, Tiplady, Walsh and Phillips (2012) state that over $90 \%$ of their sample expressed reluctance to mention their own experience of violence to a veterinarian. The reasons for this reluctance included shame about the abuse, fear of being judged by the veterinarian and fear of the consequences if the abuser found out that they had spoken about the situation.

Although these two studies were relatively small (sample sizes of 19 and 26 women, respectively), they highlight a particularly important concern regarding mandatory reporting (of suspected animal abuse): Evidence of companion animal abuse encountered at a veterinary clinic is likely to be occurring within a broader context of family violence. This violence is likely to be gender-based, and safeguarding human as well as animal welfare needs to be considered when reporting the abuse.

\section{References}

Acutt, D., Signal, T., \& Taylor, N. (2015). Mandated reporting of suspected animal harm by Australian veterinarians: Community attitudes. Anthrozoos, 28(3), 437-447.

Becker, F., \& French, L. (2004). Making the links: Child abuse, animal cruelty and domestic violence. Child Abuse Review, 13(6), 399-414. 
Febres, J., Brasfield, H., Shorey, R.C., Elmquist, J., Ninnemann, A., Schonbrun, Y.C., Temple, J.R., Recupero, P.R., \& Stuart, G.L. (2014). Adulthood animal abuse among men arrested for domestic violence. Violence Against Women, 20(9), 1059-1077.

Flynn, C.P. (2011). Examining the links between animal abuse and human violence. Crime, Law \& Social Change, 55(5), 453-468.

Hardesty, J., Khaw, L., Ridgway, M.D., Weber, D., \& Miles, T. (2013). Coercive control and abused women's decisions about their pets when seeking shelter. Journal of Interpersonal Violence, 28(13), 2617-2639.

Lachance, M. (2016). Breaking the silence: The veterinarian's duty to report. Animal Sentience 2016.006, 1-16.

Robertson, A. (2010). Legally protecting and compelling veterinarians in issues of animal abuse and domestic violence. New Zealand Veterinary Journal, 58(3), 114-120.

Simmons, C.A., \& Lehmann, P. (2007). Exploring the link between pet abuse and controlling behaviors in violent relationships. Journal of Interpersonal Violence, 22(9), 1211-1222.

Taylor, N., Signal, T., \& Stark, T. (2006). Domestic violence, child abuse and companion animal harm: Service provider perspectives. Journal of the Home Economics Institute of Australia, 13(1), 2-5.

Tiplady, C.M., Walsh, D.B., \& Phillips, C.J.C. (2012). Intimate partner violence and companion animal welfare. Australian Veterinary Journal, 90(1-2), 48-53. 